\title{
Associated antidiabetic treatment in a rural population with type 2 diabetes mellitus
}

Fernández-García, José Carlos (1), Cortés-Salazar, Carmen María (2)

1. Endocrinology Department. Virgen de la Victoria University Hospital. Málaga (Spain).

2. Badolatosa Primary Care Centre. Seville (Spain).

\section{Objectives}

To evaluate the characteristics of patients with type 2 diabetes mellitus (T2DM) attended in a rural primary care setting and to analyze associated antidiabetic treatment and metabolic control

\section{Methods}

- Cross-sectional study

- Inclusion criteria: T2DM patient followed in a primary care setting.

- Data about age, sex, body mass index (BMI), associated antidiabetic treatment, and HbA1c was collected.

Statistical analysis was performed with SPSS v 15.0 for Windows.

\section{Basal characteristics ( $\mathrm{n}-79)$}

\begin{tabular}{|l|c|}
\hline Age (years) & $70.1 \pm 11.8$ \\
Male (\%) & 63.3 \\
BMI (kg/m²) & $30.8 \pm 11.8$ \\
T2DM duration (years) & $5.9 \pm 5$ \\
Hba1c (\%) & $6.8 \pm 0.9$ \\
Triglycerides (mg/dl) & $148.3 \pm 81.1$ \\
Cholesterol $(\mathrm{mg} / \mathrm{dl})$ & $180.5 \pm 40.6$ \\
LDL-cholesterol $(\mathrm{mg} / \mathrm{dl})$ & $101.3 \pm 34.7$ \\
HDL-cholesterol $(\mathrm{mg} / \mathrm{dl})$ & $49.8 \pm 13.5$ \\
\hline
\end{tabular}

\section{Associated antidiabetic treatment (\%)}

\begin{tabular}{|c|c|}
\hline Oral Antidiabetic Drugs (OADs) & 74.7 \\
OADs + Insulin & 20.3 \\
Insulin Alone & 5 \\
\hline
\end{tabular}

\section{Distribution of antidiabetic therapy (\%)}

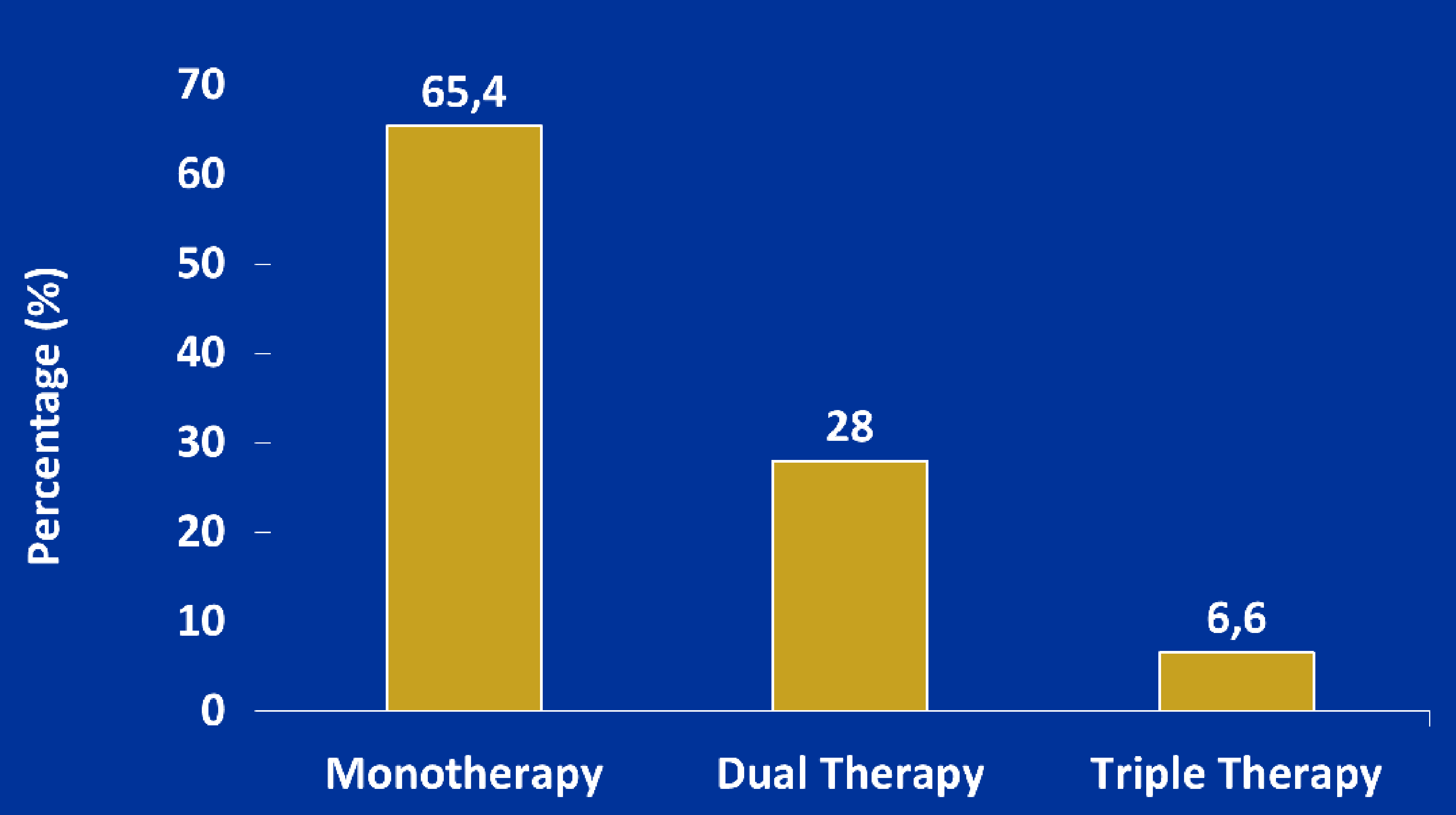

Most used drugs were metformin (85.3\%), DPP4 inhibitors (29.3\%) and sulfonilureas (20\%).

Most used insulin therapy was basal insulin + rapid-acting insulin (55\%), followed by basal alone $(35 \%)$ and pre-mixed insulins (10\%).

Mean insulin dose was 62.3 units (DE 37.1) and mean HbA1c value was 6.8\% (DE 0.9).

$\Rightarrow 61 \%$ of patients showed adequate metabolic control (defined by HbA1c $<7 \%$ ).

\section{Conclusions}

Most patients with T2DM in a rural setting are obese elderly men.

$\Rightarrow$ Antidiabetic therapy is usually based on oral drugs, being metformin the commonest drug.

Metabolic control was adequate in this group of patients, achieving more than $60 \%$ of patients $\mathrm{HbA} 1 \mathrm{c}<7 \%$. 